\title{
Les outils de mesure de la communication : regard critique sur l'état de l'art
}

\section{Maud Tixier}

\section{(2) OpenEdition}

1 Journals

Édition électronique

URL : http://journals.openedition.org/communicationorganisation/1887

DOI : 10.4000/communicationorganisation. 1887

ISSN : 1775-3546

Éditeur

Presses universitaires de Bordeaux

\section{Édition imprimée}

Date de publication : 1 novembre 1996

ISSN : 1168-5549

Référence électronique

Maud Tixier, "Les outils de mesure de la communication : regard critique sur l'état de l'art », Communication et organisation [En ligne], 10 | 1996, mis en ligne le 26 mars 2012, consulté le 10 décembre 2020. URL : http://journals.openedition.org/communicationorganisation/1887 ; DOI : https://doi.org/10.4000/communicationorganisation.1887

Ce document a été généré automatiquement le 10 décembre 2020.

(c) Presses universitaires de Bordeaux 


\title{
Les outils de mesure de la communication : regard critique sur l'état de l'art
}

\author{
Maud Tixier
}

1 La crise économique au début des années 90 a permis une Prise de conscience générale de l'importance stratégique de la communication: les dirigeants sont de plus en plus convaincus de l'importance de l'image institutionnelle, de la bonne circulation de l'information, et de la cohérence entre les communications interne et externe.

2 Les acteurs économiques demandent aujourd'hui à la communication d'être performante (d'être un facteur de l'identité et de la culture de l'entreprise, de faire passer les messages que veut diffuser l'entreprise avec le moins de déformations possible, etc.), et aussi d'aider à la prise de décisions futures au sein de l'entreprise grâce aux résultats qu'elle a obtenus elle-même.

Mais ces mêmes acteurs lui demandent surtout d'être rentable : elle doit donc s'inscrire dans une logique économique et ne plus rester à l'écart de l'exigence d'efficacité générale (un raisonnement en termes de «franc investi » n'est plus systématiquement impropre dans le domaine de la communication). Afin de s'inscrire définitivement comme l'une des facettes du management de l'entreprise, elle doit fonctionner sur un modèle managérial qui a sa logique d'efficacité et que l'on peut ainsi auditer: position de la «marque d'entreprise ", cohérence du système de marque, efficience financière de l'investissement communication... Il devient donc indispensable d'analyser les retombées d'une opération de communication et d'en évaluer l'impact (1. Tixier M., 1995). L'évaluation doit témoigner du professionnalisme de la communication, permettant ainsi à l'entreprise de mieux connaître l'image qu'elle a d'elle-même et de mieux travailler avec celle-ci, démontrant ou invalidant la rentabilité d'actions diverses de communication (2. Vidal F, 1992). L'évaluation, qui peut être qualitative ou quantitative (3. Matthys E., 1988), est un outil qui permet de mesurer le degré de réussite ou d'échec d'une opération sur différents critères. Cet outil utilise des techniques ou des méthodologies mises au point par des 
experts sur lesquelles est bâti leur savoir-faire (4. Lindenmann W., 1990) (5. Macnamara J.R., 1992).

4 Cependant, la communication est intrinsèquement un domaine qui n'est pas toujours aussi aisément évaluable que peuvent l'être le marketing ou la finance, puisque la rentabilité des actions est en partie qualitative. Pour autant, l'évaluation qui en est faite ne saurait être approximative. Celle-ci peut être de nature qualitative ou quantitative à la condition que les méthodologies utilisées soient reconnues pour leur rigueur, leur fiabilité et qu'elles aient été validées. Jusqu'à présent, des outils existent confirmant l'efficacité d'actions ponctuelles, mais il existe peu de synthèses, et encore moins d'approches globales de l'évaluation de la fonction. De plus, les méthodologies et outils permettant actuellement de mesurer la communication d'entreprise nécessitent d'être clarifiés et repensés.

Il s'agira donc ici de faire un constat des mesures existantes, en notant leurs avantages et leurs limites et les circonstances dans lesquelles leur utilisation est le plus favorable pour l'entreprise.

6 Il faut rappeler que l'enjeu de cette étude n'est pas uniquement financier mais aussi stratégique: la communication repensée peut devenir le levier d'une stratégie de croissance.

7 Dix domaines de communication ont été définis afin de mettre en valeur les méthodes de communication et outils d'évaluation propres à chacun. Il s'agit de (1) la publicité institutionnelle, (2) le lobbying et la communication d'opinion, (3) la communication de crise, (4) le design ou l'identité visuelle, (5) la communication interne, (6) la communication financière, (7) la publicité de produit, (8) la communication de recrutement, (9) le mécénat et le sponsoring, et (10) les relations de presse. Cette catégorisation permet de répertorier l'étendue de la fonction communication et d'observer les méthodes et outils propres à chaque secteur.

8 Une campagne de communication comprendra un ou plusieurs de ces domaines, qu'il faudra intégrer de façon cohérente dans une stratégie de communication inscrite dans la stratégie générale de l'entreprise (6. D'Humières P., 1993) (7. IREP, 1986).

Certains domaines de la communication refusent quasiment toute mesure ou quantification de l'impact du travail. Tel est le cas de l'industrie du design dans laquelle les professionnels estiment que la question de l'identité visuelle d'une entreprise ne devrait pas se poser en termes de prix, car le logo d'une entreprise représente de facto la plus importante source d'échange entre l'entreprise et le public.

10 En règle générale, toutefois, chaque champ de la communication dispose de son panel d'outils, et ces derniers se retrouvent souvent dans plusieurs champs différents.

\section{Méthodologie de l'étude}

11 Dans sa première phase, le projet a consisté à recenser et à analyser toutes les méthodologies et outils existants - soit usuels, soit de pointe - utilisant une méthodologie répertoriée dans chacun des domaines de la communication d'entreprise suivants: publicité institutionnelle, lobbying/communication d'opinion, communication de crise, design-identité visuelle, communication interne, communication financière, publicité de produit, communication de recrutement, mécénat-sponsoring, relations de presse, communication des dirigeants, événementiel. 

travail afin de rédiger une synthèse reflétant une approche innovante de la problématique étudiée. Chaque groupe d'enquêteurs avait à sa disposition l'ensemble des analyses de contenu effectuées par l'ensemble des groupes. L'étude de terrain a fait l'objet d'une présentation devant un panel d'experts. La discussion qui s'ensuivit a déterminé les objectifs de la synthèse.

Plusieurs axes pour cette étude ont ainsi été déterminés : (1) une typologie des mesures d'efficacité en communication; (2) l'analyse des outils classiques et des outils les plus rigoureux ou les plus sophistiqués utilisés ; (3) la distinction entre outils de pilotage et de validation avec les tendances observées vers l'un et l'autre; (4) la limite inhérente à ces tests et études; enfin (5) les facteurs déterminant le choix de la mesure d'efficacité en communication. C'est cette synthèse, présentant les résultats de l'enquête, qui vous est décrite ci-dessous.

\section{Typologie des mesures d'efficacité en communication}

Deux tendances claires apparaissent en matière d'utilisation d'outils de mesure d'efficacité. On constate tout d'abord la permanence de l'utilisation des outils "classiques", principalement dans les PME où les budgets de communication sont réduits, mais aussi dans les grands groupes où la mesure de l'efficacité en communication 
est loin d'être systématique. On discerne ensuite une volonté des grands groupes de personnaliser leurs outils en s'adressant à des agences spécialisées extérieures qui mettent au point des outils très spécifiques et performants, même s'ils sont souvent lourds à utiliser et particulièrement coûteux.

Il convient d'expliquer clairement ce qui est compris par outils «classiques » et outils «sophistiqués ». Cette différenciation, qui n'a rien de rigide et de définitif, a le mérite de mettre en lumière deux types d'approche de la communication. La première approche, relativement artisanale, aide les acteurs à se forger une opinion au jour le jour, souvent de façon intuitive, sans véritable souci d'évaluer en tant que telle l'efficacité des opérations. La seconde approche, plus professionnelle, répond à la volonté des acteurs de la communication d'être considérés comme des gestionnaires à part entière et a pour but de mesurer de façon fiable le retour sur investissement des actions de communication mises en œuvre (10. Hauss D., 1993) (11. IPRA, 1994).

Voici un tableau de critères simplifiés et de modalités d'utilisation permettant de distinguer les outils dits "classiques» des outils qualifiés de "sophistiqués». Dans ce même tableau figure également la distinction entre outils de pilotage et de validation (12. Tixier M., 1996) auxquels le texte fera référence ultérieurement.

\section{Les outils classiques}

21 Ces outils sont utilisés par toutes les entreprises, principalement dans la communication de recrutement, la communication institutionnelle et les relations avec les médias. Ce sont des outils simples et relativement peu onéreux qui, exploités rigoureusement et sur le long terme, permettent d'avoir une certaine lisibilité de la stratégie à entreprendre.

22 a) En ce qui concerne la politique de recrutement par exemple, le décompte des demandes de stage, des candidatures spontanées ou des réponses aux petites annonces est un outil fiable s'il est systématisé et toujours mis en relation avec des chiffres semblables. La comparaison de ces données avec les résultats des années précédentes ou des entreprises du même secteur constitue un indicateur correct, facile à mettre en œuvre, peu coûteux, et donc utilisé dans toutes les entreprises ayant une politique de recrutement. De la même façon, la bonne connaissance et l'image de l'entreprise chez les candidats à un poste ou par les étudiants dans un forum sont des indicateurs simples et précieux. Ces notions "intuitives" peuvent être validées par des questionnaires qui formalisent la sémiologie et les impressions.

23 b) Pour mesurer plus précisément la notoriété d'une entreprise, il existe plusieurs outils utilisés aussi bien dans des PME que dans des grands groupes. Leur coût dépend entièrement de la taille de l'échantillon, mais il peut être considérablement réduit dans les PME (public très ciblé). On étudie aussi bien la notoriété spontanée que la notoriété assistée ou le "top of mind" (première citation spontanée associant une notion ou un événement à une entreprise). Cependant, ces taux n'ont de signification qu'en comparaison avec des entreprises concurrentes ou avec les années antérieures (13. Cantorné J.-P, 1995).

Il existe par ailleurs des baromètres d'image pour mesurer l'image de l'entreprise auprès du grand public. Mais il s'agit là d'un outil plutôt complexe puisque les mesures doivent être effectuées très régulièrement, à l'aide d'un questionnaire à chaque fois identique pour ne pas biaiser les résultats. 
25 c) $85 \%$ des entreprises évaluent leurs retombées de presse (14. Roman M., 1991). Cette étude est très rarement sous-traitée (c'est le cas pour moins de $10 \%$ des entreprises). Elle s'effectue sur le plan qualitatif (ton et étude sémantique de l'article, profil des lecteurs, etc.) et sur le plan quantitatif (nombre d'articles, nombre de photos, tentative d'équivalence en achat d'espace, etc.). La limite de l'étude de la revue de presse est claire : celle-ci répertorie aussi bien les articles favorables que défavorables, sans toujours faire de distinction en termes de répercussion sur l'image.

L'efficacité de la communication se répercute également à l'intérieur de l'entreprise. Dans la plupart des entreprises, des études qualitatives sont réalisées auprès des responsables des ressources humaines, ainsi que des questionnaires sur le climat social auprès des salariés (15. Henriet B., 1992). Lorsque ces études sont très régulièrement menées, il s'agit de baromètres, comme il en existe par exemple chez ÉDF-GDF.

La plupart des entreprises cotées en bourse vérifient l'efficacité de leurs actions de communication à l'aide de critères financiers. Les critères boursiers, tels que la volatilité du titre, la durée moyenne de détention du titre et le volume de titres échangés sont des indicateurs précieux tant de la santé économique de l'entreprise que de la portée de sa communication institutionnelle, interne ou financière. Mais comme nous allons le voir, l'imbrication des phénomènes permet difficilement une analyse de l'efficacité d'un type de communication quelconque; en effet, le cours de la bourse obéit à de nombreux paramètres (financiers, économiques et politiques) en dehors des effets de la politique de communication de l'entreprise.

En revanche, ces outils d'observation ont deux avantages majeurs. Premièrement, ils ne coûtent rien puisque ces informations sont suivies avec beaucoup de soin par le service financier. Deuxièmement, ils reflètent une réalité objective, à l'inverse des questionnaires d'image. En effet, la détention de titres est une réelle marque de confiance.

Les outils dits « classiques » ne sont pas tous de simples outils d'observation. Ce sont aussi des outils d'analyse qui aident l'entreprise à prendre des décisions, à réorienter sa stratégie de communication, sa stratégie commerciale ou ses actions de sponsoring.

Pour les actions de communication événementielle (forums, salons, congrès...) comme pour les actions de mécénat, il existe une large gamme d'outils utilisés dans d'autres domaines de communication : étude de la revue de presse, fréquentation du stand dans les forums par des étudiants ou dans les salons par des clients, des soirées-spectacles par des partenaires, des assemblées générales par les actionnaires, des conférences de presse par les journalistes..., degré d'implication des divers partenaires (lecture du journal interne par les employés, nombre d'appels sur le numéro vert mis à la disposition des consommateurs, nombre de demandes de rapports annuels...), temps de passage à la télévision et audimat enregistré et, bien sûr, analyse des ventes.

31 Ces outils, même s'ils sont relativement peu fiables, au sens scientifique de ce terme, sont utilisés très couramment par les entreprises. Leurs avantages sont toujours la facilité d'utilisation, le faible coût et la rapidité des réponses.

\section{Les outils rigoureux et sophistiqués}

32 L'évolution de la communication en tant que science a encouragé l'apparition d'outils de mesure plus rigoureux qui sont aussi plus sophistiqués. Le changement des attitudes visà-vis de la nécessité de communiquer et le souci de retour sur investissement ont 
contribué à cette complexification inspirée par d'autres domaines de gestion ou par des instituts de sondage, comme le marketing, les statistiques, la modélisation mathématique, la finance, ou comme la sémiologie, la sémantique, la sociologie et la psychologie. Le développement d'outils sophistiqués est aussi fortement lié à l'essor de l'informatique, élément essentiel pour traiter les informations.

Une grande partie de ces outils très élaborés a vu le jour dans des grands groupes, et leur mise en œuvre est souvent réalisée par des agences extérieures, qui adaptent une méthodologie qui leur est propre aux exigences particulières de chaque entreprise. Ils constituent des baromètres qui donnent une vision globale des actions de communication.

Médiascore, créé par la Sofrès - institut de sondage français -, est un exemple d'outil sophistiqué; il s'agit d'un modèle mathématique combinant 200 variables interactives axées sur la sémiologie, la sémantique et la lexicographie. Cet outil peut traiter une grande variété de sujets : image politique, image de l'entreprise, nouveau produit, crise. Il peut aussi mesurer la rentabilité de la communication grâce au «scorepress d'économie » qui évalue la rentabilité financière de la communication en rapportant le «scorepress d'efficacité » au coût publicitaire des supports. Le coût d'utilisation de cette méthode varie entre 40 et $100 \mathrm{kF}$ selon la complexité de l'analyse. L'institut Synthèse crée un outil semblable à Médiascore dont les résultats relèvent les thèmes favorables et défavorables à l'entreprise.

De nombreux outils sophistiqués de prétests existent aussi. À titre d'exemple, Publitest, créé par Research Interna-tional, évalue l'impact et la compréhension d'une publicité, ainsi que l'image du produit grâce à un questionnaire. Le «tiacking» ou repérage, les courbes de réponse, et les modélisations économiques sont d'autres exemples d'outils sophistiqués fréquemment employés.

La grande limite de ces études réside dans le glissement qui s'opère sur leur objectif. En effet, le danger en communication est d'évaluer non pas l'effet d'une action, mais l'action elle-même. Ainsi, on a tendance à évaluer la campagne de publicité, son agrément, son esthétique, voire son originalité, alors que la seule question est celle de son efficacité réelle (produit-elle l'effet attendu ?). Ce glissement de l'étude de l'impact vers l'étude de l'action se vérifie dans tous les domaines de communication et montre bien la limite de la mesure de ce qui est convenu d'appeler « l'efficacité ».

\section{Les outils de pilotage et de validation}

37 Une autre classification des outils est faite en termes d'outils de pilotage et outils de validation, et l'on peut distinguer des tendances vers les uns ou les autres selon le domaine de communication.

Il semble que, dans trois domaines de la communication, l'utilisation concomitante d'outils de pilotage et de validation soit courante : la publicité de produit, la publicité institutionnelle et la communication interne.

En ce qui concerne la publicité de produit, ce fait n'a rien d'étonnant puisque c'est dans ce domaine que se sont développés les premiers outils de pilotage (16. IREP, 1988). À ses débuts, la publicité se contentait d'informer sur un produit, en décrivant ses propriétés, ou en louant ses qualités. Mais quand la publicité s'est attachée à associer une image aux produits, puis à la véhiculer, elle a très vite eu recours à des outils de pilotage, comme des 
entretiens qualitatifs ou des études sémiologiques. L'utilisation d'outils de validation est plus récente et très liée à la crise. Les publicitaires, comme les médias, ont dû, pour s'en sortir, donner des preuves à leurs clients de l'efficacité des publicités. L'utilisation d'outils, comme les posttests ou les indicateurs d'exposition, s'est largement répandue.

\section{Une tendance vers les outils de pilotage} environnement défavorable. Elle doit être rapide et ne repose donc que rarement sur l'utilisation d'outils de pilotage (18. Tixier M., 1991). En revanche, les outils de validation sont utilisés dans ce domaine. Ils peuvent être très simples (nombre de candidatures spontanées, «scorepress » ou résultats de repérages...) ou plus élaborés (étude d'image, modélisation économétrique ${ }^{1}$ ) (19. Gourieroux C et Manfort A., 1989) (20. Greene W H., 1993) (21. Judge G C., 1985).

Certains secteurs de la communication utilisent plus souvent les outils de pilotage que les outils de validation. Il s'agit de l'identité visuelle et, de manière moins forte, des relations de presse.

Dans le domaine de l'identité visuelle, de nombreux outils de pilotage sont utilisés. Des études d'image sont réalisées, afin de mieux connaître l'entreprise dont le logo doit changer. Des études sémiologiques ou des portraits chinois sont également réalisés en interne ou en externe, pour mieux appréhender l'image de l'entreprise et sa personnalité. Mais rares sont les entreprises qui testent l'efficacité d'une politique en matière d'identité visuelle. (nombre d'articles), ou plus élaborés (analyse qualitative de ces articles). Mais les entreprises se mettent de plus en plus à utiliser des outils de pilotage pour guider leurs actions en matière de presse. Comme il est nécessaire pour une entreprise d'orienter ses relations de presse, et d'ajuster ses actions en fonction de la conjoncture, les outils de pilotage prennent une importance de plus en plus grande.

\section{Une tendance vers les outils de validation}

Dans les domaines de la communication de crise et du recrutement, du lobbying et du sponsoring, les outils de validation sont largement plus utilisés que les outils de pilotage.

La communication de crise, comme son nom l'indique, est très ponctuelle et dépend d'un En matière de communication de recrutement, rares sont les entreprises qui utilisent des outils de pilotage, comme la sémiométrie ou simplement les entretiens qualitatifs avec des étudiants. En revanche, il est courant dans ce domaine de mesurer l'impact d'un forum ou de n'importe quelle autre action de communication. On peut en effet compter le nombre de demandes de stages ou de candidatures spontanées.

On peut aussi faire une analyse des contacts, à la suite d'un forum par exemple.

Le lobbying peut difficilement avoir recours à des outils de pilotage. La stratégie adoptée évolue selon les contacts obtenus et la conjoncture, mais ce ne sont pas des outils particuliers qui indiquent la nécessité de ces changements. En termes de validation. En revanche, les méthodes existent et sont simples : il suffit de voir si l'entreprise a gagné le marché qu'elle voulait ou si la loi désirée est passée (22. Farnel FJ., 1993). 
Dans le domaine du sponsoring enfin, les outils de pilotage existent. On peut avoir recours à des études sémiologiques, des entretiens qualitatifs ou même des prétests en ce qui concerne le sponsoring TV. Mais plus nombreux et plus employés sont les outils de validation : indicateurs d'exposition, de notoriété, d'image...

Cette classification reste cependant incomplète et peut même, dans certains cas, s'avérer erronée. Il est difficile de dégager une tendance vers les outils de pilotage, vu la complexité d'une politique de communication. Par ailleurs, si l'on considère que les résultats des outils de validation permettront de lancer d'autres actions de communication, chaque outil de validation joue aussi le rôle d'un outil de pilotage.

\section{Limités des tests et des études}

Même s'il est parfois facile de déterminer l'efficacité d'une mesure de communication (par exemple, si à la suite d'une action de lobbying, des lois sont modifiées, un amendement passé ou un appel d'offre obtenu), ces cas sont assez peu fréquents, car les outils dont nous avons parlé ont tous leurs limites.

51 Tout d'abord, il faut souligner qu'il est très difficile d'évaluer les effets de plusieurs actions menées de front. Pour le lobbying, toute évaluation d'action est partielle et peut être remise en cause par un événement extérieur qui peut surgir à tout instant, dans l'environnement de l'entreprise ou de l'organisation concernée (23. Le Grelle B., 1988). De même, une communication, par exemple financière, ne peut en aucun cas être l'unique facteur déterminant une action observable. Une entreprise qui réussit une privatisation a probablement eu une bonne communication financière, mais la conjoncture externe et la situation interne de l'entreprise ont certainement aussi eu un rôle déterminant pour le succès (24. Doyen M., 1989).

Il est donc difficile de prendre de tels indicateurs, financiers ou autres, comme facteurs exclusifs de la qualité de la communication, par exemple financière. Même si l'on ne peut nier l'existence d'indicateurs permanents de la qualité de la communication, notamment financière, leur évolution obéit à de nombreux paramètres. D'ailleurs, une évaluation post test de privatisation montre qu'à la question « où avez-vous cherché des informations ?» les réponses sont floues et la décision d'investissement a des origines complexes. Chaque média joue un rôle, mais il est impossible d'en connaître le degré d'importance. De plus, dans ce cas précis, deux facteurs ne sont pas mesurables: le réseau bancaire et la conjoncture internationale.

Le domaine de la publicité de produit est aussi très sensible à ce type de problème. Il dispose fréquemment d'une part prépondérante des dépenses de communication de l'entreprise, et il est donc très préoccupant qu'on ne puisse pas distinguer les effets de deux actions simultanées - ceux d'une publicité de produit ou d'une promotion, par exemple (25. Guelfand G., 1995).

4 De même qu'une observation peut être le résultat de plusieurs actions menées simultanément, un même individu peut appartenir à plusieurs cibles à la fois: le professionnel, le consommateur moyen, le père de famille, le sportif-Comment savoir ce qui a réellement influencé les opinions de l'individu? On peut constater un changement après une campagne sans pour autant être sûr de l'origine. 

l'environnement politique de l'entreprise. Si un dirigeant refuse les conclusions d'une étude, peu importe la performance de l'outil; il existe encore des directeurs de communication qui ne tiennent pas compte de ces outils ou qui refusent simplement de les utiliser, sous prétexte que la communication n'est pas un phénomène rationnel. Cette part de psychologie humaine est l'essence même de la communication.

d'analyse de retombées médiatiques (chez Framatome par exemple), ont leurs inconvénients : malgré les performances de l'informatique, l'évaluation des relations de presse reste avant tout une analyse de contenu effectuée par l'homme et assistée par l'ordinateur. Il y a donc inévitablement une part de subjectivité dans l'analyse, aussi faible soit-elle.

D'autre part, même si l'on utilise des outils d'évaluation, on n'est jamais à l'abri d'un échec, lié à la conjoncture ou à l'imprévisibilité des facteurs humains. Ainsi, La Poste avait vraiment bénéficié de son implication dans la voile. En créant un équipage de postiers, lors d'une des plus grandes régates internationales, elle avait joui d'une image compétitive, courageuse, innovante. Mais la deuxième participation a été un échec aussi complet qu'inattendu ${ }^{2}$; et la Poste n'a plus jamais renouvelé son engagement dans le domaine de la voile...

61 Certains phénomènes psychologiques échappent aussi totalement aux études. Alors qu'au moment des prétests pour le lancement de la Twingo de Renault, cette voiture semblait adaptée aux jeunes et aux conducteurs citadins, elle est aujourd'hui, de manière totalement imprévue, vendue aussi aux personnes d'âge plus mûr de manière non négligeable.

L'étude des différents outils d'évaluation en communication permet aussi de constater que jamais les investissements humains ne pourront faire l'objet de mesures aussi rigoureuses que les investissements matériels. En ce qui concerne les politiques de recrutement par exemple, aucun outil n'évalue le manque à gagner sur un candidat à fort potentiel qui n'a pas été touché ou qui a préféré rejoindre un concurrent.

Effectivement, quoi de plus difficile que d'évaluer un retour sur investissement humain? Et par voie de conséquence, que signifie une communication de recrutement efficace? De 
la même façon, le coût du lobbying est extrêmement difficile à évaluer, si l'on veut y incorporer le temps passé par les responsables des actions de lobbying.

Lié à cette question de l'évaluation des coûts est le problème de la continuité des évaluations. La mise en œuvre d'une stratégie de lobbying, par exemple, doit normalement faire l'objet d'un contrôle permanent et d'une évaluation périodique de son adéquation et de ses résultats. La démarche qui suit l'évolution des actions permet de débusquer les erreurs et d'empêcher un grand écart du but recherché. Un manque de recours aux techniques de contrôle du lobbying peut entraîner des résultats graves. Nombreuses sont les instances où un manque de réflexion sur l'impact d'une intervention de lobbying a coûté cher pour la défense d'un projet et pour les grands contrats des entreprises. En règle générale, on note qu'une certaine régularité est essentielle pour nombre de ces opérations. Le baromètre pour déceler les évolutions internes prévues ou imprévues dans l'entreprise ou un secteur d'activité ne sera utile que s'il est utilisé régulièrement.

Toute étude n'a de valeur que par comparaison; les études de communication interne n'ont aucune signification en soi ; leurs résultats doivent être comparés à des données antérieures et replacés dans un contexte précis. Il ne suffit pas de savoir si le climat social de l'entreprise est bon, mais s'il est meilleur qu'il ne l'était précédemment, afin de voir s'il y a eu des progrès et si la communication interne est efficace : c'est le différentiel qui a une signification, pas le score brut. Pour les baromètres donc, les exigences se corsent. Il faut reprendre les mêmes questions, au mot près et dans le même ordre. Ils doivent aussi être pensés dès leur conception pour du long terme, au risque sinon de perdre toute valeur (26. Downs N. J., 1991).

Ceci ne devrait pas être un problème en soi, mais une évaluation périodique et des comparaisons fréquentes ne font qu'augmenter le coût de la démarche.

Les tests se font soit immédiatement après une campagne, soit à intervalles réguliers (baromètres), soit en permanence (repérage ou "tracking») (27. Riley R., 1994). Si, comme nous venons de le noter, la continuité et le suivi sont importants, il n'en demeure pas moins que des tests trop fréquents manquent de sensibilité à court terme (les échantillons n'étant en général guère supérieurs à cent personnes), et représentent un coût élevé (1 MF par an).

Enfin, nous pouvons nous demander - ceci à un tout autre niveau - s'il faut toujours tester. Effectivement, à trop vouloir tester, on bride la créativité. Pour reprendre l'exemple de la publicité institutionnelle, nous notons que les prétests présentent l'inconvénient de supprimer l'impact de la campagne en voulant y faire adhérer un maximum de personnes. Il faut nécessairement prendre des risques et faire un choix d'ordre stratégique.

69 Enfin, certaines études, même très fiables, sont souvent trop coûteuses par rapport à l'investissement. Cela peut être le cas du mécénat ou du sponsoring. Dans ces cas, il paraît parfaitement illogique de dépenser plus en test qu'en communication.

\section{Facteurs déterminant le choix de la mesure d'efficacité en communication}

Devant la multitude d'outils, l'entreprise doit se donner des points de repère et des critères de choix. L'outil n'est en aucun cas une fin en soi, mais un moyen au service de la 
stratégie générale de communication de l'entreprise ou de l'institution. L'outil retenu doit donc être bien adapté à l'action de communication évalué, et doit aussi répondre à de nombreux critères.

\section{La taille de l'entreprise}

71 La taille de l'entreprise est un facteur déterminant dans le choix des études. Ce sont essentiellement les grosses entreprises et les grands groupes internationaux qui commandent les études les plus spécialisées en raison de leur degré d'implication et de leur engagement financier. Il est évident que les PME et PMI n'ont pas le même budget que les grandes entreprises. Par voie de conséquence, les fonds qui sont alloués à la communication ne permettent souvent pas l'emploi d'outils trop complexes. Il faut rappeler qu'une étude efficace, fiable et sérieuse, commandée à l'extérieur, coûte généralement autour de $100 \mathrm{kF}$. Dans le domaine de la publicité de produit par exemple, un «buy test » coûte de 80 à $100 \mathrm{kF}$, un « tracking » coûte 1 MF par an... Dans les grandes entreprises, près de $4 \%$ du budget d'une action de communication est alloué aux études. On imagine alors quelle doit être l'ampleur d'une action de communication, pour qu'au moins $100 \mathrm{kF}$ soient consacrés aux études.

La tendance pour les PME est donc surtout aux outils simples de validation, mais l'on peut se demander si elle ne serait pas aux outils de pilotage si ces derniers étaient moins chers.

Dans les grosses entreprises, la tendance est généralement double : on utilise à la fois les outils de pilotage et les outils de validation. Par leurs actions, les grandes entreprises participent même à l'élaboration d'outils sophistiqués et performants, en faisant part de leur attentes aux concepteurs.

\section{Le secteur d'activité}

74 Le secteur d'activité est aussi un élément non négligeable dans le choix d'outils. Les entreprises qui effectuent le plus d'études sont celles pour lesquelles l'image joue un rôle prépondérant, comme c'est le cas dans les milieux bancaires et financiers, là où les services proposés font la différence. Pour construire un imaginaire et une identité, elles ont recours à tous les domaines de la communication. Les mesures sont ainsi à la hauteur des investissements : complètes, précises et régulières. Il faut en effet veiller sans cesse à l'image et la réorienter. Une grande importance est accordée aux mesures comparatives qui permettent, comme les cartes perceptuelles ou « mapping », de se situer par rapport à la concurrence : comment adopter un positionnement distinctif et fort dans l'esprit du public dans un secteur très encombré. Ces remarques sont valables aussi pour tout secteur très compétitif où il faut s'affirmer et se distinguer en termes d'image, comme par exemple dans les compagnies aériennes.

Les secteurs d'activité qui doivent lutter contre des a priori négatifs et critiques développent aussi les études. C'est le cas du secteur de la chimie ou du nucléaire : il s'agit d'informer, de se rapprocher du public et de refléter des valeurs dignes. Rhône-Poulenc évalue ainsi son sponsoring TV, et Eli encadre sa politique autour de l'effort et du dépassement avec l'aide de la sémiométrie. Comme il faut aussi sensibiliser le public à son action et aux normes de sécurité, l'efficacité des relations publiques et du lobbying n'est pas négligée. 


\section{La structure, élément d'environnement}

La structure du capital influe sur la politique de communication de l'entreprise et par conséquent sur les moyens qu'elle se donne pour l'évaluer. Ainsi, lorsque l'entreprise est constituée par actions, elle se doit de communiquer avec ses actionnaires et de les traiter en tant qu'interlocuteurs privilégiés. Elle développe donc l'événementiel et les relations de presse. Les mesures de fréquentation et les indicateurs d'exposition jouent un rôle particulier. Une entreprise à structure familiale, en revanche, effectue souvent moins d'études de pilotage car de nombreuses décisions sont prises sans véritable consultation, notamment en matière de sponsoring, où les souhaits et avis du conseil d'administration, et plus particulièrement du chef familial, sont pris en compte. Il n'y a alors pas lieu de mesurer l'efficacité d'une action de communication qui ne répond pas à des objectifs clairement fixés et définis en amont.

\section{Le contexte économique et social}

Le contexte économique et social influence évidemment aussi les choix d'outils de l'entreprise. Aujourd'hui, la crise économique frappe encore durement nombre de pays, qui se débattent dans des problèmes d'inflation, de chômage ou d'endettement, problèmes qui rejaillissent sur la stabilité de leur monnaie. La consommation des ménages reste relativement faible, y compris dans les pays qui connaissent une reprise économique. C'est donc dans ce contexte particulier, mouvant et fragile, que les entreprises placent leurs politiques de communication. Il s'agit de prendre toute la mesure des changements en cours, de discerner les évolutions conjoncturelles des mutations structurelles et d'adapter la politique de communication à ces mutations, dans le respect de la stratégie de l'entreprise.

Les exemples de la communication de recrutement et de la communication interne en période de chômage sont pertinents. Comment recruter sans heurter de front les sensibilités alors qu'on vient de licencier 200 personnes? Telle est la question que se posent à l'heure actuelle de nombreuses entreprises. Les changements de valeurs ont incité les entreprises à diversifier leurs outils de communication pour en accroître l'efficacité : ainsi, nombreuses sont celles qui ont développé une approche hors média (salons, forums, visites d'entreprise...).

Dans un contexte d'environnement changeant, les outils de pilotage et les prétests apparaissent comme des outils majeurs d'évaluation: ils ne doivent pas chercher à prévoir l'efficacité, mais à éviter les erreurs, à confirmer ou infirmer une idée ou une orientation, alors que les outils de validation ne valident qu'a posteriori les actions menées. Dans cet esprit, les actions les plus efficaces sont celles dont on a mesuré, avant de les lancer, l'impact sur une longue durée, en prenant bien soin de tenir compte des évolutions de mentalités, de comportements, de jugements ou encore de perceptions du public.

\section{Le facteur temps}

Enfin, on n'insistera jamais assez sur l'importance du facteur temps dans l'évaluation de l'efficacité de la communication. Pour se révéler efficace dans la durée, la communication doit s'inscrire sur un fil continu d'actions souvent discrètes. L'important sera de toujours déterminer des moyens et un contenu, une expression et des outils justes et créatifs. La 
publicité institutionnelle, qui a pour but d'accroître la notoriété et d'améliorer l'image de l'entreprise en transformant une réalité économique abstraite en une entité à dimension humaine intéressante, s'inscrit aussi forcément dans le long terme. Rompre la continuité d'une telle action reviendrait à en perdre tous les bénéfices accumulés. En revanche, la communication de crise nécessite une mobilisation rapide, et en principe de courte durée.

La contrainte du temps peut aussi influencer les méthodes de communication que peut choisir l'entreprise. L'étude d'image, par exemple, constitue un outil complet qui permet d'évaluer la communication de crise, mais il y a un inconvénient de coût et de temps puisqu'il faut élaborer un questionnaire fiable, recueillir les informations et les synthétiser, alors que le temps est une contrainte clé. Bien sûr, le temps détermine aussi quel genre de communication sera utilisé pour atteindre un objectif donné : en temps de crise économique, on va au plus pressé : on vise d'abord la promotion des produits, plutôt que d'investir dans l'institutionnel.

En résumé, il faut concevoir l'intervention de la communication comme un instrument important de médiatisation et de reconnaissance des choix de l'entreprise ou de toute autre institution en externe comme en interne. Savoir où va l'entreprise, dans quel paysage elle va s'inscrire, quelle position elle veut y tenir et comment elle peut le justifier sont les préalables à toute stratégie de communication. En effet, une communication qui ne porte pas le dessein de l'entreprise perd une partie de son potentiel et de son efficacité.

La fonction communication est donc destinée à aider l'entreprise à atteindre ses objectifs économiques et stratégiques dans un environnement parfois hostile et instable. La communication s'inscrit désormais dans une logique économique et doit répondre à des critères de gestion et de productivité. Par ailleurs, l'efficacité de la communication dépend de la compréhension et de l'adéquation de cette politique à la stratégie de l'entreprise. Mais s'il est indispensable d'expliciter la stratégie, il n'est pas forcément souhaitable de l'exprimer directement.

La communication est donc au service de la stratégie de l'entreprise. Cela a nécessairement une influence sur la décision de mesurer ou non l'impact d'une action de communication. En effet, dès lors que la communication devient stratégique, il devient du même coup stratégique de pouvoir en mesurer les retombées.

$\mathrm{Du}$ point de vue de la réflexion méthodologique, il faudra toujours rappeler les limites auxquelles se heurte une telle démarche. On l'a vu, il n'est en effet pas toujours simple de mesurer l'efficacité en communication, car des raisons d'ordre méthodologique, budgétaire, politique, commercial peuvent réduire à néant l'espoir d'évaluer les résultats des différentes communications de l'entreprise et leur valeur ajoutée. Cependant, il est indéniable que mesurer cette efficacité permettra à la communication d'entreprise d'atteindre cette envergure managériale à laquelle elle aspire. 


\section{BIBLIOGRAPHIE}

CANTORNE J.-P., Évaluation de l'efficacité publicitaire, Paris, FCB, Département études et recherches, 1995, (13).

D'HUMIERES P., Management de la communication d'entreprise, Eyrolles, 1993, (6).

DOYEN M, Communication financière, Paris, Éditions d'Organisation, Paris, 1989, (24).

DOWNS N.J., Audit instrumentation in organisational communication, Australian Communication Review, vol. XII, 1991, (26).

D'UNRUG M.-C, Analyse de contenu et acte de parole : de l'énoncé à l'enonciation, Paris, Éditions Universitaires, 1974, (9).

FARNEL F.-J., Le lobbying : stratégies et techniques d'intervention, Paris, Éditions d'Organisation, 1993, (22).

GOURIEROUX C. et MANFORT A., Statistiques et modèles économétriques, Paris, Economica, 1989, (19).

GREENE W.-H., Econometric analysis, Mc Millan, 1993, (20).

GUELFAND G., Efficacité publicitaire ou publicité efficace, Paris, IREP, 1995, (25).

HAUSS D., Measuring the impact of public relations, Public Relations Journal, 1993, (10).

HENRIET B., « Audit de la communication interne », Personnel, n 332, Paris, 1992, (15).

IPRA (International Public Relations Association), «Quality customer satisfaction, public relations : new directions for organisational communication », Gold Paper n 10, P \& P Gmbh, Allemagne, 1994, (11).

IREP, Identité et image d'entreprise, Paris, 1986, (7).

IREP, Mesurer l'efficacité de la publicité, Paris, 1988, (16).

JUDGE G.C, The theory and practice of econometrics, New-York, Wiley, 1985, (21).

LE GRELLE B., Profession lobbyman : le pouvoir des coulisses, Paris, Hachette, 1988, (23).

LINDENMANN W., «Research, evaluation and measurement : a national perspective », Public Relations Review, 1990, (4).

MACNAMARA J.R., «Evaluation in public relations : the Achilles heel of the public relations profession », International Public Relations Review, vol. XV, n 4, 1992, (5).

MATTHYS E., Du quantitatif au qualitatif, Paris, IREP (Institut de recherches et d'études publicitaires), 1988, (3).

MUSNIK I., « Peut-on réellement évaluer l'efficacité des relations presse ? », CB News, n 361, Paris, 1994, (17).

RILEY R., Comment utiliser le tracking pour améliorer l'efficacité de la communication. Enseignements et évaluation de la méthode, Paris, IREP, 1994, (27).

ROMAN M., La perception des médias, des publics et des programmes à travers les études qualitatives, Paris, IREP, 1991, (14). 
TIXIER M., La communication de crise : enjeux et stratégies, coordinateur et chapitre de conclusion, Mc Graw Hill, Coll. Nouveaux Concepts, Nouvelles Idées de communication, Paris, juin 1991, (18).

TIXIER M., Evaluation/mesures d'efficacité en communication, Chaire « communication et management ", ESSEC, Paris, 1995, (1).

TIXIER M., « La mesure de la communication : réalité ou utopie ? », Faits de Sociétés, n² 28, Paris, 1995, (8).

TIXIER M., « L'attitude des entreprises face au choix des outils : pilotage ou validation ? » Faits de Sociétés, $\mathrm{n}^{\circ}$ 32, Paris, 19, (12).

VIDAL F., Efficacité quand tu nous tiens, CB News, n² 268, 1992, (2).

\section{NOTES}

1. Par exemple en communication de crise, se reporter à l'étude Évaluation/ mesure d'efficacité en communication de crise. La modélisation économétrique, p. 17, réalisée à l'ESSEC, avril 1995.

2. Tout d'abord, il n'y avait plus que des postiers sur le bateau. L'image de l'entreprise « combattante et prête à tout » en a été amoindrie. Et suite à une bagarre en Uruguay, certains membres de l'équipage se sont retrouvés en prison.

\section{RÉSUMÉS}

La fonction communication, pour être une fonction managé-riale reconnue, doit se prouver utile et rentable. Elle exige le développement et le perfectionnement d'outils et de méthodes tendant à prouver cette efficacité et cette rentabilité. C'est de ces outils, de leurs avantages et de leurs limites, ainsi que des circonstances dans lesquelles ils peuvent être utilisés, que traite cet article.

The communication function today finds itself at a threshold: though acknowledged as being essential, it is still not fully recognized as a managerial function. It thus needs to develop tools testing its efficiency and profitability. This article will seek to evaluate these tools, and the circumstances in which they are used.

\section{INDEX}

Mots-clés : Outils, méthodologie, communication, évaluation

\section{AUTEUR}

\section{MAUD TIXIER}

Maud Tixier est professeur à l'ESSEC depuis 1972. Elle est titulaire de la chaire « communication et management ». Elle possède un doctorat en Sciences de l'Information et de la communication du Celsa (Paris IV-Sorbonne). Elle a enseigné aux USA, au Canada 
et en Grande-Bretagne et a été professeur visitant à la Manchester Business School en Grande-Bretagne. Elle a notamment publié les livres suivants : Travailler en Europe, aux éditions Liaisons en 1992, La Communication de crise, chez McGraw Hill en 1991 (multiauteurs), et La Négociation commerciale, aux Éditions ESF en 1988 (avec Alain Jolibert). Ses principaux centres d'intérêt sont la communication corporate, le recrutement et la mobilité des dirigeants en Europe, la communication interculturelle, la rédaction/ conception publicitaire, la communication des entreprises sur le web. 